\title{
Picosecond Photovoltaic Response in Tilted Lanthanum Doped Manganite Films
}

\author{
Zhiqing Lu, ${ }^{1}$ Hao Ni, ${ }^{1}$ Jianfeng Xi, ${ }^{1}$ Xiaoming Li, ${ }^{2}$ and Kun Zhao ${ }^{1}$ \\ ${ }^{1}$ College of Science, China University of Petroleum, Beijing 102249, China \\ ${ }^{2}$ Department of Physics, The Chinese University of Hong Kong, Hong Kong \\ Correspondence should be addressed to Hao Ni; nihona@163.com
}

Received 24 January 2013; Revised 7 August 2013; Accepted 9 September 2013

Academic Editor: Bhushan Sopori

Copyright (c) 2013 Zhiqing Lu et al. This is an open access article distributed under the Creative Commons Attribution License, which permits unrestricted use, distribution, and reproduction in any medium, provided the original work is properly cited.

\begin{abstract}
Anisotropic picosecond photovoltaic responses were observed in lanthanum doped manganite $\mathrm{La}_{x} \mathrm{Ca}_{1-x} \mathrm{MnO}_{3}(x=0.67$ and 0.4$)$ thin films, which were deposited on miscut $\mathrm{LaSrAlO}_{4}$ substrates under ultraviolet pulsed laser irradiation without external bias. The $10 \%-90 \%$ rise time and the full width at half maximum of $\mathrm{La}_{0.67} \mathrm{Ca}_{0.33} \mathrm{MnO}_{3}$ were 470 and 585 ps, respectively, and those of $\mathrm{La}_{0.4} \mathrm{Ca}_{0.6} \mathrm{MnO}_{3}$ were 220 and 515 ps. The photovoltage sensitivities of $\mathrm{La}_{0.67} \mathrm{Ca}_{0.33} \mathrm{MnO}_{3}$ and $\mathrm{La}_{0.4} \mathrm{Ca}_{0.6} \mathrm{MnO}_{3}$, which are sensitive to the concentrations of lanthanum of the samples, are $0.28 \mathrm{~V} / \mathrm{mJ}$ and $3.47 \mathrm{~V} / \mathrm{mJ}$, respectively. The photosensitivity in the films deposited on $\mathrm{MgO}$ is higher than that in those deposited on $\mathrm{LaSrAlO}_{4}$ substrates, for it has a big lattice mismatch. These results should open a route for the application of lanthanum doped manganite as an ultrafast photodetector material.
\end{abstract}

\section{Introduction}

Previously, the photovoltaic effect was reported in the perovskite-based $\mathrm{p}-\mathrm{n}$ junctions, thin films, and single crystals [16]. The charge carriers of $p-n$ junctions are separated by the electric field in the space charge region, and the photovoltage can be observed. However, as a typical photodetector structure, $\mathrm{p}-\mathrm{n}$ junction detectors cannot normally have ultrafast responses, which would limit the applications in nanosecond and picosecond pulse laser detections. Recently, focus on tilted structures in lanthanide doped perovskite oxides which present ultrafast photoresponses and high sensitivities in photodetection [7-9]. Theoretical researches demonstrated that ultrafast photoresponse processes should be attributed to anisotropy diffusion and Seebeck effect. Researches also demonstrated that the concentrations of lanthanide of the samples could modulate the photoresponse signals in nanometer size domain tilted thin films, which indicate a new type nanometer scale photosensitive source.

In the present study, we deposited different concentrations of lanthanum doped manganite thin films, $\mathrm{La}_{0.67} \mathrm{Ca}_{0.33}$ $\mathrm{MnO}_{3}$ (LCMO1) and $\mathrm{La}_{0.4} \mathrm{Ca}_{0.6} \mathrm{MnO}_{3}$ (LCMO2), on miscut $\mathrm{LaSrAlO}_{4}$ (LSAO) substrates, and observed picosecond ultraviolet (UV) photovoltaic effect under pulsed laser irradiations without external bias. The photoresponse signals are sensitive to the measurement circuit input impedance. A small paralleling resistance with thin film can effectively improve the response speed. When the $\mathrm{La}_{x} \mathrm{Ca}_{1-x} \mathrm{MnO}_{3}$ (LCMO) thin film is connected in parallel with a $0.5 \Omega$ resistance, the $10 \%-90 \%$ rise time (RT) and the full width at half maximum (FWHM) decrease to $470 \mathrm{ps}$ and $585 \mathrm{ps}$ for LCMO1, and $220 \mathrm{ps}$ and $515 \mathrm{ps}$ for LCMO2. The voltage sensitivities can reach $0.28 \mathrm{~V} / \mathrm{mJ}$ for $\mathrm{LCMO} 1$ and $3.47 \mathrm{~V} / \mathrm{mJ}$ for $\mathrm{LCMO} 2$, respectively. Correspondingly, the current sensitivities are $0.21 \mathrm{~A} / \mathrm{mJ}$ for LCMO1 and $3.44 \mathrm{~A} / \mathrm{mJ}$ for LCMO2. The comparative experiments on LCMO film deposited on miscut MgO substrates prove that the photo sensitivity is higher in a big lattice mismatch system. All these experimental results reveal that the lanthanum doped manganite films on miscut substrates can be used as ultrafast photodetective materials.

\section{Experimental}

The $100 \mathrm{~nm}$ thick LCMO thin films were deposited on miscut LSAO substrates with (001) LSAO plane tilted to the surface wafer at an angle $\alpha$ of $10^{\circ}$ by facing-target sputtering technique from stoichiometry targets [10]. During the deposition, the substrates were kept at $680^{\circ} \mathrm{C}$, with oxygen partial pressure of 
30 mTorr. The film thickness, controlled by sputtering time with the deposition rate, is $100 \mathrm{~nm}$. After the deposition, the vacuum chamber was immediately backfilled with $1 \mathrm{~atm}$ oxygen gas to improve the oxygen stoichiometry. X-ray diffraction (XRD) was adopted to characterize the structure of the as-deposited products.

For the photovoltaic measurements, two Ag electrodes $\left(1 \times 5 \mathrm{~mm}^{2}\right.$ in size $)$ separated by $3 \mathrm{~mm}$ were prepared on the surface of the LCMO, as shown in Figure 2(b). Electrodes "A" and "B" are parallel to the tilted direction, while "C" and " $D$ " are perpendicular to the tilted direction. Thus, the photovoltage of the "AB" and "CD" can be investigated and characterized, and $V_{\mathrm{AB}}$ and $V_{\mathrm{CD}}$ can represent the photovoltage of tilted direction and untilted direction, respectively. The third harmonic of an actively and passively mode-locked Nd: yttriumaluminum-garnet laser was used as the source at room temperature. The laser was operated at the wavelength of $355 \mathrm{~nm}$ (3.49 eV photon energy) with $25 \mathrm{ps}$ duration at $10 \mathrm{~Hz}$ repetition rate, and the energy density was $0.0127 \mathrm{~mJ} / \mathrm{mm}^{2}$. Thus, the on-sample energy was $0.435 \mathrm{~mJ}$. The photovoltaic signals were monitored by a Tektronix sampling oscilloscope with a $2.5 \mathrm{GHz}$ bandwidth terminated into $1 \mathrm{M} \Omega$ and $50 \Omega$.

\section{Results and Discussion}

The microstructure of LCMO/LSAO sample with different lanthanum component was checked with X-ray diffraction (XRD) $\theta-2 \theta$ scan as shown in Figure 1(a), where the [001] axis was aligned carefully and the offset point was set by $10^{\circ}$ to satisfy Bragg's diffraction. Except for the (00l) LSAO substrate and the $(h 0 h)$ LCMO film reflections, there was no diffraction peak from impurity phase or randomly oriented grain, indicating the epitaxial growth of the sample. In LCMO thin epitaxial film, the decrease of lanthanum component led to a consequence of lattice contraction due to the transition from $\mathrm{Mn}^{3+}$ to $\mathrm{Mn}^{4+}$. Therefore, the diffraction peak moved to a higher angle.

The linear current-voltage $(I-V)$ characteristics, shown in Figure 2(a), of the LCMO films indicated ohmic contacts between the films and Ag electrodes. And the resistivity of the LCMO1 film is larger than that of the LCMO2 film. The opencircuit photovoltaic pulses between the two electrodes which were painted on the surface of the LCMO were observed without any applied bias when the LCMO surfaces were irradiated by the pulse laser directly. Figure 3 shows typical photovoltaic responses of LCMO thin films along the tilted direction, recorded by the oscilloscope terminated into $1 \mathrm{M} \Omega$ and $50 \Omega$. When the impedance of the oscilloscope was set as $1 \mathrm{M} \Omega$, the peak voltages $V^{\mathrm{P}}$ was $0.053 \mathrm{~V}$ for the LCMO1 and $0.661 \mathrm{~V}$ for the LCMO2, respectively. However, it should be noted that the responses are composed of a fast rise time, several oscillations, and a slow decay. The RC constant in the circuit and the impedance mismatch should be responsible for these phenomena. In order to reduce the influence of the $\mathrm{RC}$ effect in the measurement, we changed the import impedance of the oscilloscope from $1 \mathrm{M} \Omega$ to $50 \Omega$. As shown in Figure 3, the RT and the FWHM decreased to 870 ps and $2435 \mathrm{ps}$ for the LCMO1, and $278 \mathrm{ps}$ and $1624 \mathrm{ps}$ for

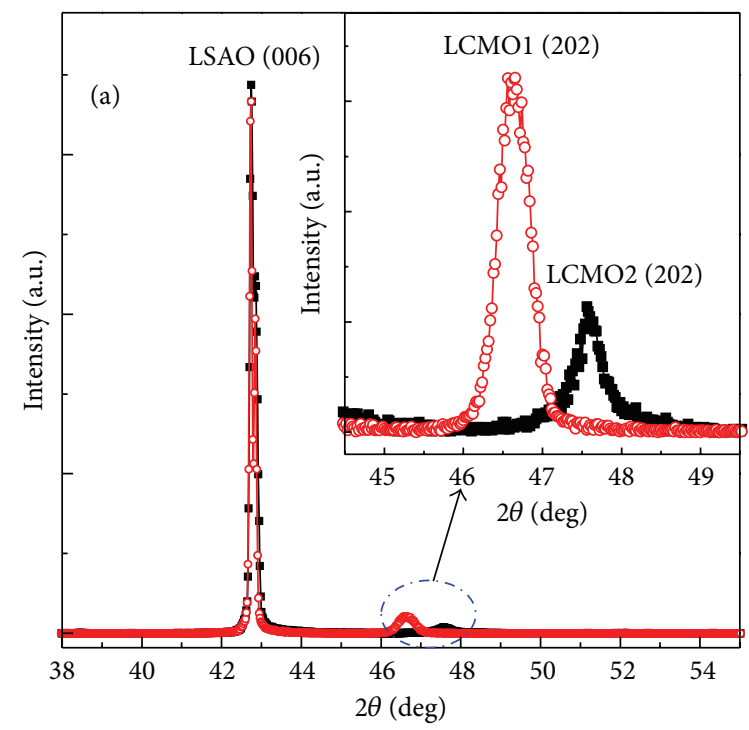

FIGURE 1: XRD patterns of LCMO films on miscut LSAO substrates. The inset shows the details of the LCMO peaks. Except for the $(00 l)$ LSAO substrate and the $(h 0 h)$ LCMO film reflections, there was no diffraction peak.

the $\mathrm{LCMO} 2$. In addition, a $0.5 \Omega$ resistance was connected in parallel with the LCMO thin film, and the RT and the FWHM are 470 ps and 585 ps for LCMO1, and 220 ps and 515 ps for LCMO2, indicating that the photovoltaic response of the LCMO thin film is a picosecond process.

The anisotropic photoelectric effect was systematically investigated along the tilted direction and untilted direction. And the waveforms which were recorded by the oscilloscope with the input impedance of $50 \Omega$ were shown in Figures 3 and 4 . For the titled direction, the peak photovoltage $V_{\mathrm{AB}}{ }^{\mathrm{P}}$ in LCMO1, which is exhibited in Figure 3(a), is $0.0294 \mathrm{~V}$ when the film surface was irradiated directly. At the untitled direction, $V_{\mathrm{CD}}{ }^{\mathrm{P}}$ (shown in Figure 4(a)) in LCMO1 is $0.002 \mathrm{~V}$, which is 14 times smaller than $V_{\mathrm{AB}}{ }^{\mathrm{P}}$. In LCMO2, the peak photovoltages $V_{\mathrm{AB}}{ }^{\mathrm{P}}$ and $V_{\mathrm{CD}}{ }^{\mathrm{P}}$ are $0.542 \mathrm{~V}$ and $0.0308 \mathrm{~V}$, respectively, shown in Figures 3(b) and 4(b). It is noteworthy that the photovoltaic response was reversed when the LSAO substrate was irradiated. Therefore, the whole photoresponse process can be understood as follows. The ultrafast laser pulses irradiated the LCMO film surface uniformly and generated photoinduced charge carriers (electron and hole pairs) with gradient distribution $\left(d n_{i} / d z\right)$ perpendicular to the film surface [9]. Due to anisotropy and the large ratio of $\rho_{[101]} / \rho_{(101)}$, the diffusion current along $[10 \overline{1}]$ axis is much larger than that along the [101] axis. In carriers' diffusion process, the mobility of electrons is much larger than that of holes $\left(\mu_{n}-\mu_{p}>0\right)$, and electron-hole pairs were separated and generated the diffusion current density

$$
J_{y} \approx J_{[10 \overline{1}]} \cos \alpha \approx k_{0} T \Delta \mu_{[10 \overline{1}]} \sin 2 \alpha \frac{\left(d n_{i} / d z\right)}{2} .
$$

From Figure 3, we can deduce that the voltage sensitivities are $0.28 \mathrm{~V} / \mathrm{mJ}$ for tilting $\mathrm{LCMO} 1$ and $3.47 \mathrm{~V} / \mathrm{mJ}$ for tilting 


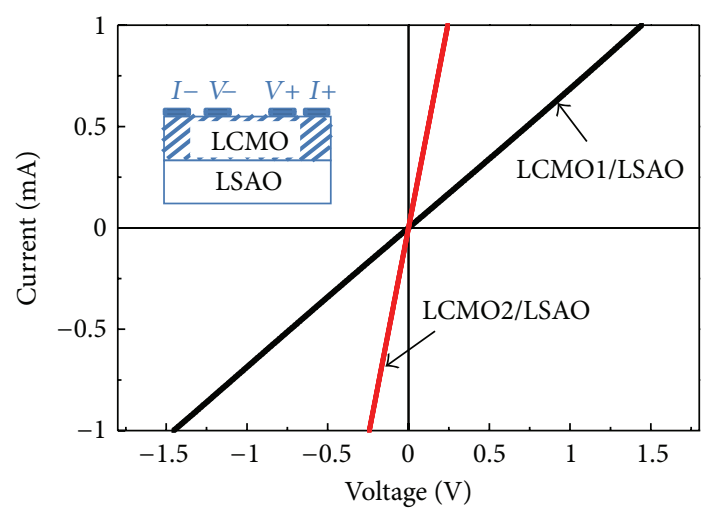

(a)

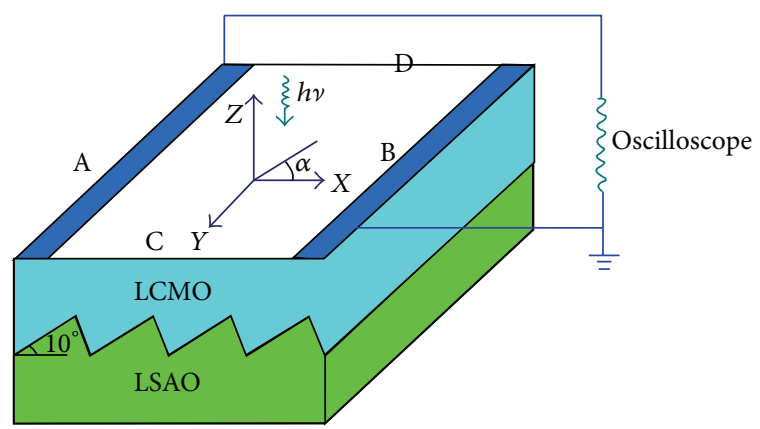

(b)

FIGURE 2: (a) $I-V$ characteristics of the LCMO1 and LCMO2 films along tilted directions (AB) at room temperature. (b) Schematic illustration of the samples, electrodes structure, and the circuit of the measurements. Electrodes A and B are along the tilted direction ( $x$ is the "tilted direction" of the film) and electrodes C and D are along the untilted direction ( $y$ is the "untilted direction" of the film).

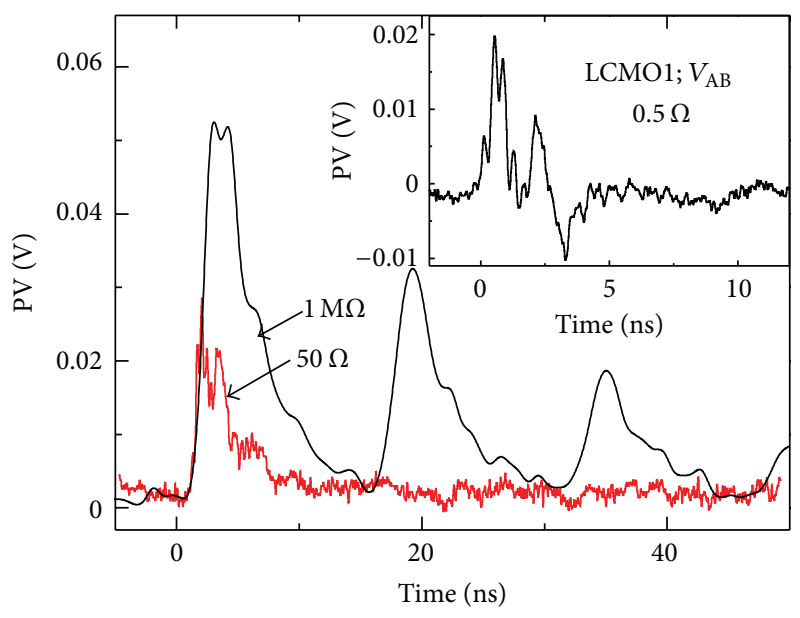

(a)

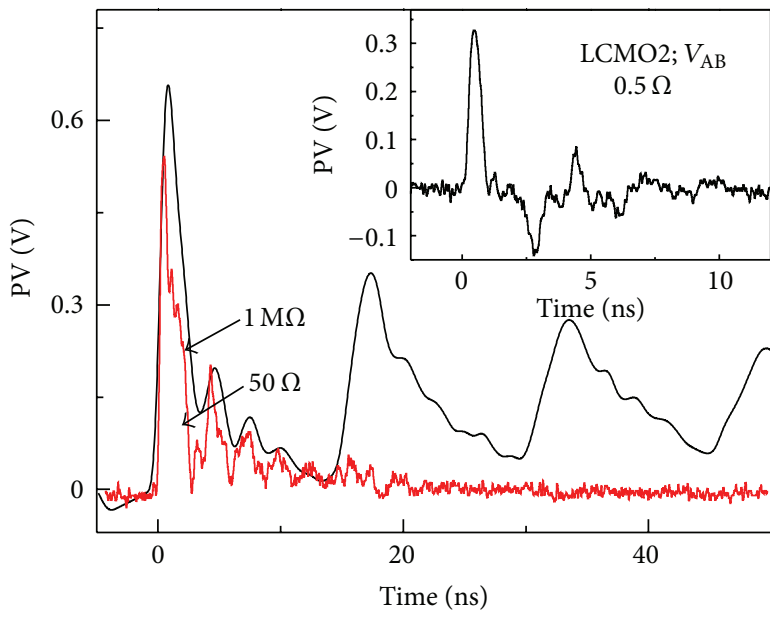

(b)

FIGURE 3: Photovoltaic response of (a) LCMO1 film and (b) LCMO2 film, under the illumination of a 355 nm laser pulse in duration of 25 ps along the tilted direction, recorded by an oscilloscope terminated into $1 \mathrm{M} \Omega$ and $50 \Omega$. The inset shows the photovoltaic response with a $0.5 \Omega$ resistance which was connected in parallel with the LCMO thin film.

LCMO2. Similarly, we can get the current sensitivities which are $0.21 \mathrm{~A} / \mathrm{mJ}$ for tilting LCMO1 and $3.44 \mathrm{~A} / \mathrm{mJ}$ for tilting LCMO2. These experimental results show that the photoinduced voltage effect in the tilting LCMO films is not only an ultrafast process but also highly sensitive to the UV laser pulse. The photosensitivity of LCMO2 was higher than that of LCMO1. In further study, we also deposited LCMO1 and LCMO2 films on miscut $\mathrm{MgO}$ substrates (at a tilt angle of $10^{\circ}$ ) and observed the ultrafast photovoltaic responses in LCMO/ $\mathrm{MgO}$ samples [9]. All the experiment conditions were the same as before. We can see clearly that the photosensitivity in $\mathrm{LCMO} 2 / \mathrm{MgO}$ was higher than that in LCMO1/MgO. And the LCMO films on $\mathrm{MgO}$ substrates showed much higher photosensitivity than the same component content LCMO films on LSAO substrates.

To understand the mechanism, high resolution transmission electron microscope (HRTEM) analysis of the interface of the LCMO/LSAO and LCMO/MgO has been done. From the images by HRTEM, we can clearly see that there is a good epitaxial growth in the LCMO/LSAO sample, due to the small lattice mismatch [11]. However, there exist a lot of dislocations and domains in $\mathrm{LCMO} / \mathrm{MgO}$, due to the larger lattice mismatch. Dislocations and domain boundaries consist of a lot of dangling bonds, which can create a lot of carriers under UV irradiation. Thus, LCMO deposited on MgO substrates with large lattice mismatch shows higher photovoltaic sensitivity.

\section{Conclusion}

In summary, we deposited lanthanum doped manganite thin films on miscut LSAO substrates and observed anisotropic picosecond UV photovoltaic responses under pulsed laser irradiation without external bias. A $0.5 \Omega$ resistance was connected in parallel with the $\mathrm{La}_{1-x} \mathrm{Ca}_{x} \mathrm{MnO}_{3}$ (LCMO) thin film. And the RT and the FWHM decreased to 470 ps and 585 ps for LCMO1, and 220 ps and 515 ps for LCMO2. The voltage 


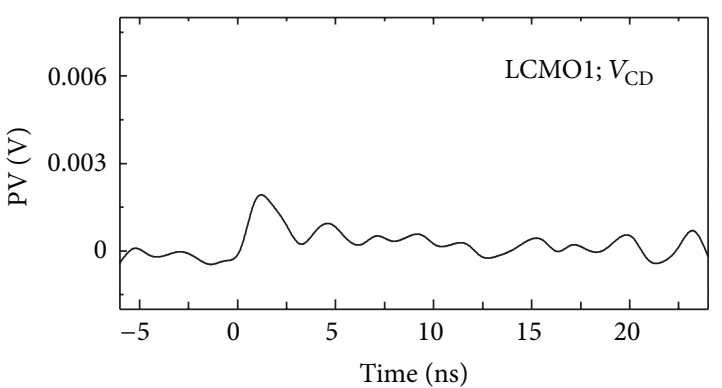

(a)

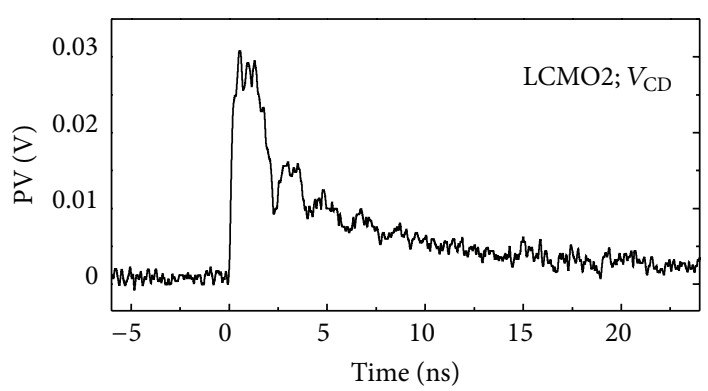

(b)

FIGURE 4: Photovoltaic responses of (a) the LCMO1 film (b) the LCMO2 film, under the illumination of a $355 \mathrm{~nm}$ laser pulse along the untilted direction $\left(V_{\mathrm{CD}}\right)$, recorded by the oscilloscope terminated into $50 \Omega$.

sensitivities reached $0.28 \mathrm{~V} / \mathrm{mJ}$ for $\mathrm{LCMO} 1$ and $3.47 \mathrm{~V} / \mathrm{mJ}$ for LCMO2. The current sensitivities are $0.21 \mathrm{~A} / \mathrm{mJ}$ for LCMO1 and $3.44 \mathrm{~A} / \mathrm{mJ}$ for LCMO2. From the above experiments, we can see several significant characteristics of the photovoltaic effect of the lanthanum doped manganite thin film: (I) the photovoltage along the tilted direction $\left(V_{\mathrm{AB}}{ }^{\mathrm{P}}\right)$ is larger than that along the untilted direction $\left(V_{\mathrm{CD}}{ }^{\mathrm{P}}\right)$; (II) the peak value of photoresponse in LCMO2 is higher than that in LCMO1; (III) the photosensitivity in LCMO films deposited on $\mathrm{MgO}$ is higher than that deposited on LSAO substrates, for the LCMO films deposited on $\mathrm{MgO}$ have a big lattice mismatch. These results should carve out a new way for the application of LCMO as an ultrafast photodetector material.

\section{Acknowledgments}

This paper has been supported by National Key Basic Research Program of China (2013CB328706), special-funded program on national key scientific instruments and equipment development (2012YQ14005), Beijing National Science Foundation (4122064), and Science Foundation of China University of Petroleum (Beijing) (QZDX-2010-01 and KYJJ2012-06-27). The authors express their thanks for the samples prepared by Professor. Hong-Kuen Wong and Mr. Yu-Chau Kong in The Chinese University of Hong Kong.

\section{References}

[1] Y. G. Zhao, J. J. Li, R. Shreekala et al., "Ultrafast laser induced conductive and resistive transients in $\mathrm{La}_{0.7} \mathrm{Ca}_{0.3} \mathrm{MnO}_{3}$ : charge transfer and relaxation dynamics," Physical Review Letters, vol. 81, no. 6, pp. 1310-1313, 1998.

[2] M. Rajeswari, C. H. Chen, A. Goyal et al., "Low-frequency optical response in epitaxial thin films of $\mathrm{La}_{0.67} \mathrm{Ca}_{0.33} \mathrm{MnO}_{3}$ exhibiting colossal magnetoresistance," Applied Physics Letters, vol. 68, no. 25 , pp. 3555-3557, 1996.

[3] J. R. Sun, C. M. Xiong, B. G. Shen, P. Y. Wang, and Y. X. Weng, "Manganite-based heterojunction and its photovoltaic effects," Applied Physics Letters, vol. 84, no. 14, pp. 2611-2613, 2004.

[4] H.-B. Lu, K.-J. Jin, Y.-H. Huang et al., "Picosecond photoelectric characteristic in $\mathrm{La}_{0.7} \mathrm{Sr}_{0.3} \mathrm{MnO}_{3} / \mathrm{Si}$ p-n junctions," Applied Physics Letters, vol. 86, no. 24, Article ID 241915, 3 pages, 2005.
[5] H. Katsu, H. Tanaka, and T. Kawai, "Photocarrier injection effect on double exchange ferromagnetism in ( $\mathrm{La}, \mathrm{Sr}) \mathrm{MnO}_{3} /$ $\mathrm{SrTiO}_{3}$ heterostructure," Applied Physics Letters, vol. 76, no. 22, pp. 3245-3247, 2000.

[6] H.-U. Habermeier, X. H. Li, P. X. Zhang, and B. Leibold, "Anisotropy of thermoelectric properties in $\mathrm{La}_{2 / 3} \mathrm{Ca}_{1 / 3} \mathrm{MnO}_{3}$ thin films studied by laser-induced transient voltages," Solid State Communications, vol. 110, no. 9, pp. 473-478, 1999.

[7] K. Zhao, K.-J. Jin, H. Lu et al., "Transient lateral photovoltaic effect in p- $\mathrm{n}$ heterojunctions of $\mathrm{La}_{0.7} \mathrm{Sr}_{0.3} \mathrm{MnO}_{3}$ and Si," Applied Physics Letters, vol. 88, no. 14, Article ID 141914, 2006.

[8] H. Ni, D. Yu, K. Zhao et al., "Oxygen content dependence of the photovoltaic characteristic of miscut manganite thin films," Journal of Applied Physics, vol. 110, no. 3, Article ID 033112, 2001.

[9] H. Ni, K. Zhao, K. J. Jin et al., "Nano-domain orientation modulation of photoresponse based on anisotropic transport in manganite films," Europhysics Letters, vol. 97, no. 4, Article ID 46005, 2012.

[10] X. T. Zeng and H. K. Wong, "Epitaxial growth of single-crystal (La, Ca) $\mathrm{MnO}_{3}$ thin films," Applied Physics Letters, vol. 66, no. 24, pp. 3371-3373, 1995.

[11] X. M. Li, K. Zhao, H. Ni et al., "Voltage tunable photodetecting properties of $\mathrm{La}_{0.4} \mathrm{Ca}_{0.6} \mathrm{MnO}_{3}$ films grown on miscut $\mathrm{LaSrAlO}_{4}$ substrates," Applied Physics Letters, vol. 97, no. 4, Article ID 044104, 2010. 

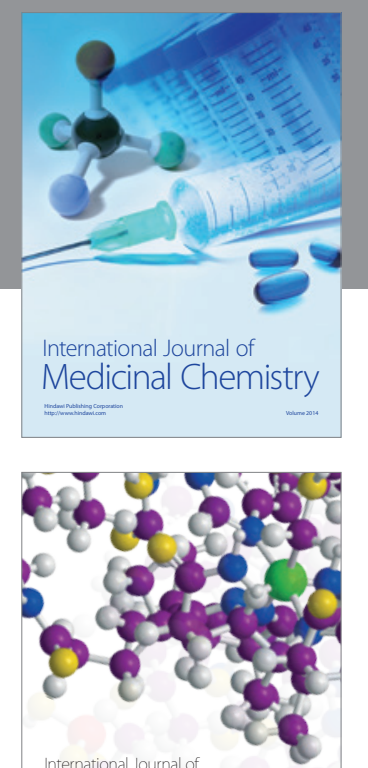

\section{Carbohydrate} Chemistry

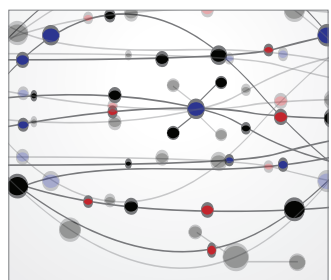

The Scientific World Journal
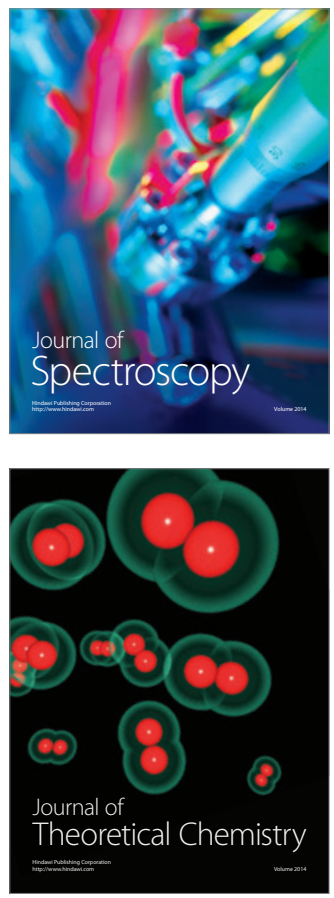
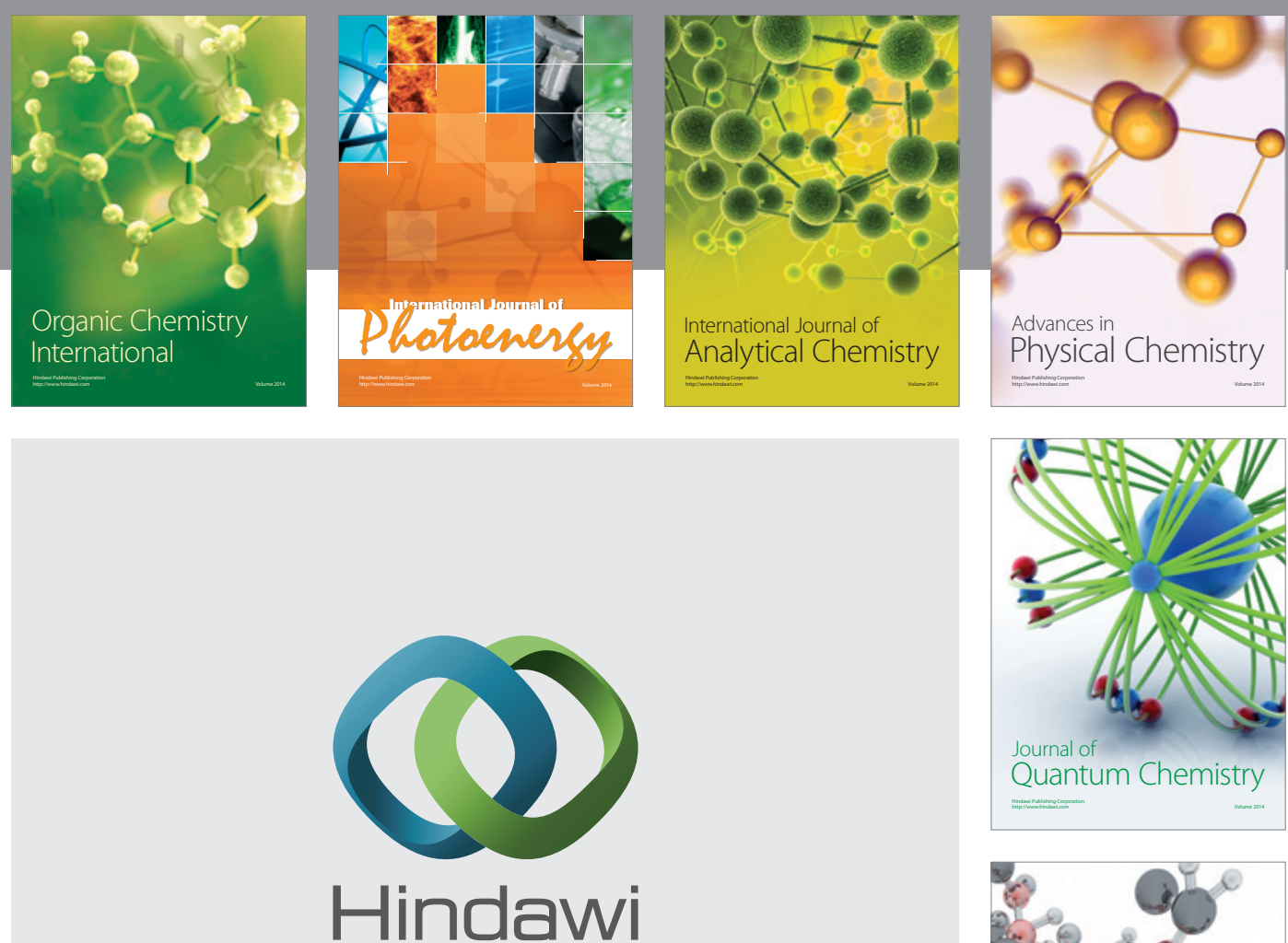

Submit your manuscripts at

http://www.hindawi.com

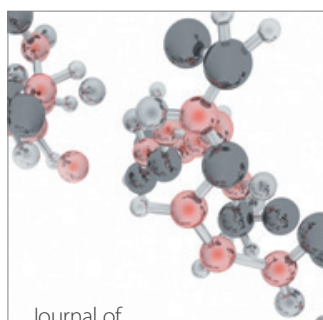

Analytical Methods

in Chemistry

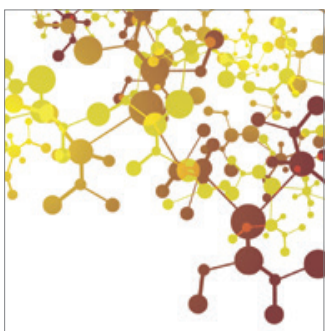

Journal of

Applied Chemistry

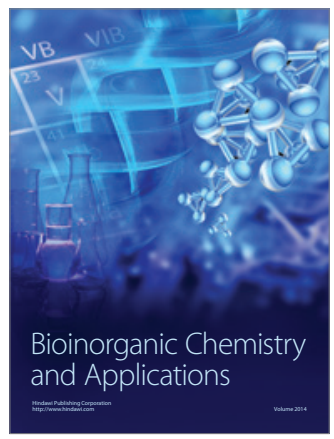

Inorganic Chemistry
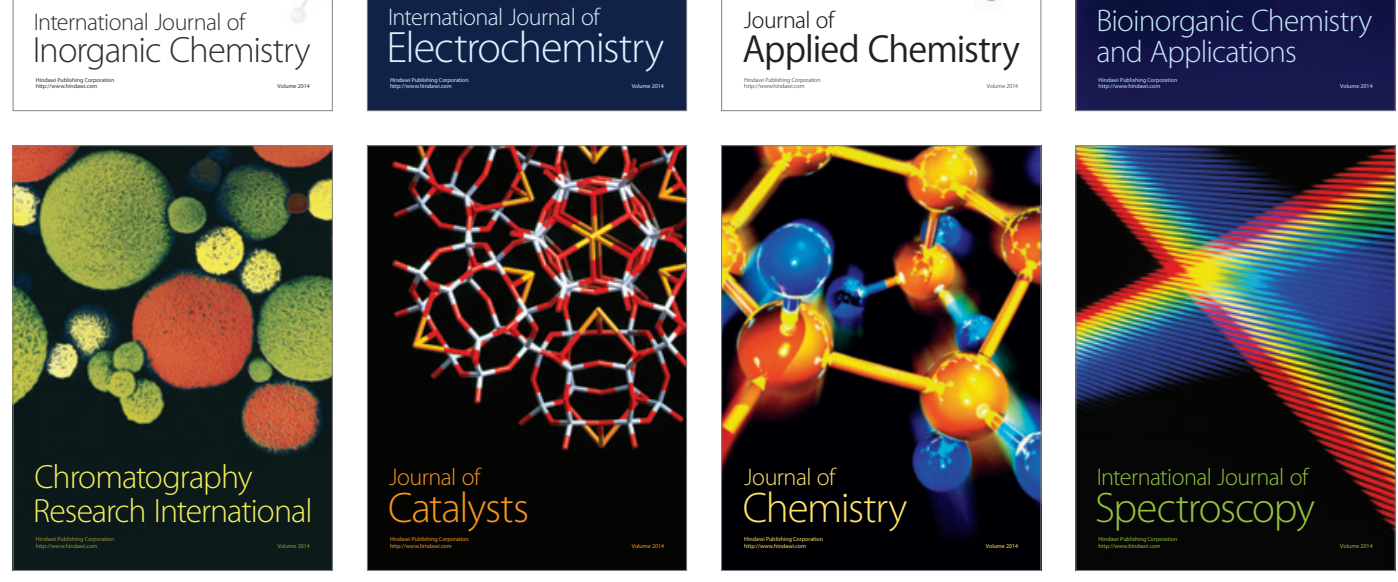